\title{
ELECTROSTATIC AND SPECIFIC BINDING TO MACROMOLECULAR
}

\section{LIGANDS. A GENERAL ANALYTICAL EXPRESSION FOR THE} DONNAN VOLUME

Encarna Companys(a), Josep Lluís.Garcés(a), José Salvador(a), Josep Galceran(a), Jaume Puy(a) and Francesc Mas*(b).

(a) Departament de Química, Universitat de Lleida (UdL). Av. Rovira Roure, 191. E-25198 Lleida (SPAIN, EU)

(b) Centre especial de Recerca de Química Teòrica (CeRQT, PCB) and Departament de Química Física. Facultat de Química, Universitat de Barcelona (UB). C/ Martí i Franquès, 1. E-08028 Barcelona (SPAIN, EU).

* corresponding author

\section{Abstract}

The dependence of the apparent acid dissociation constant of charged polyacids on the ionic strength and on the degree of dissociation is analysed in terms of the Donnan model. We introduce a new approximate analytical expression for the Donnan volume which depends on the ionic strength and on the degree of dissociation with only one adjustable parameter. This expression stems from analytical solutions of the non-linear Poisson-Boltzmann equation (NLPB) under different geometries. Two kinds of polyelectrolytes are studied: polyacrylic acid (PAA), as a model of homogeneous ligand, and Aldrich Humic acid (HA), a heterogeneous ligand which exhibits a $\mathrm{p} K_{\mathrm{a}}$ dependent on the $\mathrm{pH}$, even when the polyelectrolytic correction is applied. The new approximate Donnan volume expression allows to justify the empirical dependence of previous expressions found in the literature on the ionic strength, gives rise to master curves well behaved in all the range of macromolecular charges and can fit experimental results in good agreement with classical treatments like NLPB, used as a reference, involving a 
greatly simplified numerical treatment. Three different complexation isotherms are independently used to account for the specific binding. The robustness of the results is evidenced by the fact that similar central moments of the affinity distribution underlying each isotherm and of the adjustable parameter of the Donnan volume are obtained in each case.

Keywords: macromolecular complexation, polyelectrolytic effects, specific binding, polyacrylic acid, humic acid, Donnan volume, Natural Organic Matter

\section{Introduction}

The binding to Natural Organic Matter (NOM) is a relevant topic in the circulation of ions in environmental systems. Since acid-base ionisable groups participate in the binding sites of NOM, two components have been recognized in the binding energy: one of electrostatic origin, giving rise to the electrostatic or "atmospherical" binding, and a second chemical component responsible for the specific binding [1].

The electrostatic binding renders the apparent constants dependent on $\mathrm{pH}$ and ionic strength, $I$, the so-called polyelectrolytic effect. As a first approximation, mean field theories, mainly the non-linear Poisson-Boltzmann equation (NLPB) [2,3], have been used to account for the electrostatic binding. Different approximate models have been developed in order to avoid the numerical solution of the NLPB equation. Some are based on the assumption of a small deviation of the electroneutrality inside a certain volume [4,5]; Counterion Condensation model (CC) [6,7] proposes that, in cylindrical geometry, when the charge spacing along a rod of infinite length increases above a critical level, counterions "condense" ("territorial binding") depending on the dielectric constant of the solvent and on the charge of the counterions [8-10]. Another approximate model (the Donnan approach, D) is based on a biphasic concept: polyions are immersed in separate permeable gel phases. This approach assumes complete electroneutrality inside and outside the permeable gel phase, and the potential difference across the semipermeable membrane of the gel phase (identified as the surface potential, or Donnan 
potential) is then obtained from Donnan equilibrium conditions [11,12]. In some cases, it is difficult to obtain reliable measurements of the volume of this gel phase and empirical expressions for the Donnan volume have been reported for different polyions. The Donnan volume increases as $I$ decreases, and a linear relationship in the log-log scale has been suggested for the humic matter [13]. However, the results obtained are not fully satisfactory in all the range of macromolecular charges.

Moreover, when different chemical functionalities or interactions between sites are present, the specific binding energy is not a constant but has to be described by a discrete or a continuous distribution function [14-16]. It has been shown that different affinity distributions can reproduce the binding data at wide intermediate coverage ranges with similar accuracy [15], so that results between different assumed distributions have to be compared in terms of the moments of the distribution.

Both, the electrostatic and specific bindings are coupled, since the macromolecular charge depends on the specific binding, which at the same turn, depends on the concentration of complexing agents at the macromolecular surface (ruled by the polyelectrolytic effect). Thus, both, the set of parameters characterizing such distribution of affinities and the set describing the polyelectrolytic correction, have to be simultaneously fitted from experimental data in a general procedure.

It is the aim of the present paper to check the use of a new analytical expression for the Donnan volume in the description of the proton binding to two different kinds of ligands: the polyacrylic acid (PAA), as an example of homogeneous polyelectrolyte, and the humic acid (HA), as an example of heterogeneous polyelectrolyte. The new expression for the Donnan volume can be deduced from a combination of the Donnan ideas and the NLPB results aiming at describing a wide range of macromolecular charges, ionic strengths and geometries. Actually, present results will be compared with the NLPB equation, used as a reference approach (see appendix A). 


\section{Theoretical background}

Acid-base equilibria of polyions are generally described by the Henderson-Hasselbach equation

$$
\mathrm{p} K_{\text {app }}=-\log c_{\mathrm{H}}-\log \left(\frac{\alpha_{\mathrm{d}}}{1-\alpha_{\mathrm{d}}}\right)
$$

where $\mathrm{p} K_{\text {app }}$ indicates the apparent dissociation constant of the polyacid, and $\alpha_{\mathrm{d}}$ is the degree of dissociation of the macromolecule.

Splitting the binding energy into an intrinsic or specific chemical component, $-R T \log K_{\mathrm{c}}$, and an electrostatic work, which within the mean field approximation can be written as $F \psi_{\mathrm{S}}, K_{\text {app }}^{-1}$ can be factorized as

$$
\mathrm{p} K_{\text {app }}=\log K_{\mathrm{c}}-\frac{1}{\ln 10} \frac{F \psi_{\mathrm{S}}}{R T}
$$

where $\psi_{\mathrm{S}}$ is the electrostatic potential close to the binding sites with respect to the bulk solution (which is used as the origin), $F$ is the Faraday constant, $R$ is the gas constant, $T$ the temperature. $K_{\mathrm{c}}$ is also called average equilibrium function [1,17] and measures the chemical or specific binding energy so that

$$
\log K_{\mathrm{c}}=-\log c_{\mathrm{H}_{\mathrm{s}}}+\log \left(\frac{1-\alpha_{\mathrm{d}}}{\alpha_{\mathrm{d}}}\right)=-\log c_{\mathrm{H}_{\mathrm{s}}}+\log \left(\frac{Q_{\max }-Q}{Q}\right)
$$

where $Q$ and $Q_{\max }$ are the charge and the maximum charge in the fully deprotonated macromolecule and $c_{\mathrm{H}_{\mathrm{S}}}$ labels the concentration of protons in a volume element close to the binding sites of the polyelectrolyte. 


\subsection{Specific binding}

Both $K_{\mathrm{c}}$ and the coverage, $\theta=\frac{Q_{\max }-Q}{Q_{\max }}$, can be related to the distribution of affinities present in the heterogeneous macromolecules, the so called affinity spectrum, $\mathrm{p}(\log k)[16]$. In the simplest case, $\theta$ is a weighted addition of local Langmuir complexation isotherms

$$
\theta=\int_{-\infty}^{\infty} p(\log k) \frac{1}{1+10^{-(\log k+\log c)}} d \log k
$$

where $c$ is the concentration in the vicinity of the macromolecule. Results obtained using different isotherms to fit binding data cannot be compared in terms of the isotherm parameters since the physical meaning and the dimensions of these parameters are specific of each isotherm. However, they can be compared by computing the moments of the respective affinity distributions underlying each isotherm. Actually, it has been shown [15] that the values of $\log K_{\mathrm{c}}$ at intermediate coverages are almost just function of the average, $\mu$, and the standard deviation, $\sigma$, of the affinity spectrum

$$
\begin{aligned}
& \mu=\langle\log k\rangle \\
& \sigma^{2}=\left\langle(\log k)^{2}\right\rangle-\mu^{2}
\end{aligned}
$$

and independent from the particular shape of the distribution for unimodal distributions. Thus, one expects to find an average affinity and variance almost independent of the isotherm used in the fitting of the specific binding for interemediate coverages, while divergent values of these parameters would be obtained when very low or very high coverage is included in the experimental binding data. Table 1 reports the relationships between the parameters and the moments of the underlying distributions for the complexation isotherms used in this work: Langmuir-Freundlich, Frumkin and uniform. 


\subsection{Donnan model for the electrostatic binding}

In the Donnan approach, the polyelectrolyte is considered to behave as an electrically neutral gel phase having a particular volume, $V_{\mathrm{D}}$, throughout which there is a uniform averaged electrostatic potential known as the Donnan potential, $\psi_{\mathrm{D}}$, with respect to the uniform zero potential of the solution phase. Neglecting the influence of the osmotic pressure, the equilibrium condition between phases reads $[18,19]$

$$
\frac{a_{j, \mathrm{D}}}{a_{j}}=\left(\frac{a_{\mathrm{H}, \mathrm{D}}}{a_{\mathrm{H}}}\right)^{z_{j}}
$$

where $j$ indicates the species, $z_{j}$ its ionic charge and $a_{j}$ labels its activity at the bulk solution.

Under equilibrium conditions, the electrochemical potential of the ion should be the same in both phases. Thus for the proton,

$$
a_{\mathrm{H}, \mathrm{D}}=a_{\mathrm{H}} \exp \left[\frac{-F \psi_{\mathrm{D}}}{R T}\right]
$$

The exponential term can be easily interpreted since $F \psi_{\mathrm{D}}$ is the approximate work to bring a mole of protons from the bulk solution to the Donnan phase.

Equations (7) and (8) provide the Donnan potential, $\psi_{\mathrm{D}}$, if a suitable model for the activity coefficients is assumed.

Unfortunately, models for the activity coefficient of $j$ inside the Donnan phase are rare. In the simplest case, the activity coefficient of all ions is assumed to be the same at the bulk solution and the Donnan phase, recovering from (8) the Boltzmann equation

$c_{j, \mathrm{D}}=c_{j} \exp \left(-\frac{z_{j} F \psi_{\mathrm{D}}}{R T}\right)=c_{j} \exp \left(-z_{j} \tilde{\psi}_{\mathrm{D}}\right)$

where the tilde labels the dimensionless potential, $\tilde{\psi}_{\mathrm{D}} \equiv F \psi_{\mathrm{D}} / R T$

On the other hand, the electroneutrality condition of the gel phase prescribes

$Q / V_{\mathrm{D}}+\sum z_{j}\left(c_{j, \mathrm{D}}-c_{j}\right)=0$ 
where $Q$ is the net charge of the polyion, $V_{\mathrm{D}}$ is the Donnan gel volume, $c_{j, \mathrm{D}}$ is the concentration of component $j$ with charge $z_{j}$ (including sign) in the Donnan volume and $c_{j}$ is its concentration in the bulk.

The use of (9) in (10) determines the Donnan potential if $V_{\mathrm{D}}$ is known over the complete range of solution conditions. This strategy has been widely applied $[13,20]$. Experimental techniques, like viscosimetry and diffusion light scattering [13,21,22], have been used to estimate specific gel volumes. Not always do these measurements manage to agree between one another [21]. Operational Donnan volumes are also widely used. In this way, an expression for $V_{\mathrm{D}}$ is assumed and its parameters are determined using the merging of $Q v s . \mathrm{pH}$ curves at different ionic strengths into a unique "master curve", $Q$ vs. $\mathrm{pH}_{\mathrm{D}}$ (being $\left.\mathrm{pH}_{\mathrm{D}} \equiv-\log c_{\mathrm{H}, \mathrm{D}}\right)$ as a criterion.

Benedetti et al. [13] also showed that the apparent Donnan volume of humic matter decreases with increasing ionic strength. A linear relationship was suggested when plotted on a log-log scale

$\log V_{\mathrm{D}}=b(1-\log I)-1$

where coefficient $b$ varies with the type of humic substance. However, this Donnan volume sometimes leads to high Donnan corrections at low macromolecular charges that can hardly be realistic.

\section{A new expression for the Donnan volume}

Keeping the simplicity of the Donnan methodology, we propose an expression for the Donnan volume based on combining the ideas of the Donnan model with the results of NLPB. In this way, we expect to obtain a very general Donnan volume expression able to reproduce NLPB results in a wide range of polyelectrolytes, homogeneous or heterogeneous, linear or branched, various geometries (planar, cylindrical or spherical, and probably "intermediate" geometries between these 3) and charge densities.

The starting point is the combination of the electroneutrality equation, Eqn. (10) with Eqn. (9) as 


$$
V_{\mathrm{D}}=\frac{z Q}{2 I \sinh \left(z \tilde{\psi}_{\mathrm{D}}\right)}
$$

where $z$ is the valence of the symmetric background electrolyte. The key point consists in identifying the Donnan potential, $\tilde{\psi}_{\mathrm{D}}$ with the dimensionless surface potential given by the NLPB equation, $\tilde{\psi}_{\mathrm{S}}$. As the electrostatic work is written $z \tilde{\psi}$, with $\tilde{\psi}=\tilde{\psi}_{\mathrm{S}}$ in NLPB and $\tilde{\psi}=\tilde{\psi}_{\mathrm{D}}$ in the Donnan model, with this identification we will be looking for a Donnan potential that leads to the same electrostatic binding work than that corresponding to the NLPB description.

Once this identification is assumed, relationships between surface charge and surface potential available in the literature for different geometries within the NLPB approach can be used in (12) to work out expressions for the Donnan volume in terms of the macromolecular charge and ionic strength.

\subsection{Planar geometry}

When the polyion is modelled as an infinite planar surface, the exact relationship between the surface charge and the surface potential for symmetric electrolytes is given by the well-known equation [23]:

$$
\frac{Q}{A}=\frac{\sqrt{8 \varepsilon R T I}}{z} \sinh \left(\frac{z \tilde{\psi}_{\mathrm{S}}}{2}\right)
$$

where $Q / A$ is the charge density on the surface of total area $A$.

The definition of $a=1 / A \sqrt{8 \varepsilon R T}$, allows to convert (13) into

$$
\sinh \left(\frac{z \tilde{\psi}_{\mathrm{S}}}{2}\right)=\frac{a Q z}{\sqrt{I}}
$$

Then, if we replace $\sinh \left(z \tilde{\psi}_{\mathrm{D}}\right)$ by $\sinh \left(z \tilde{\psi}_{\mathrm{S}}\right)=2 \sinh \left(\frac{z \tilde{\psi}_{\mathrm{S}}}{2}\right) \sqrt{1+\sinh ^{2}\left(\frac{z \tilde{\psi}_{\mathrm{S}}}{2}\right)}$ in eq (12) and we use (14), we obtain a new expression for the Donnan volume given by: 


$$
V_{\mathrm{D}} \simeq \frac{1}{4 a \sqrt{I+a^{2} Q^{2} z^{2}}}
$$

The units for the parameter $a$ will depend on the units of the charge of the polyelectrolyte. $a Q$ has the dimensions of $\left(\mathrm{mol} \mathrm{L}^{-1}\right)^{1 / 2}$. For PAA, the charge $Q$ is given in $\mathrm{mol} \mathrm{L}^{-1}$, then, the parameter $a$

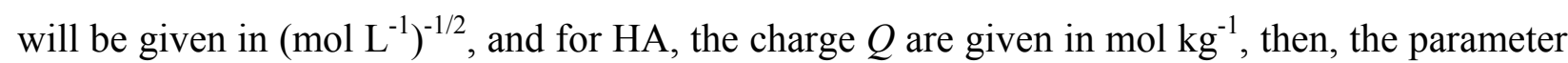
$a$ will be given in $\mathrm{kg}(\mathrm{mol} \mathrm{L})^{-1 / 2}$.

\subsection{Cylindrical and spherical geometries}

As it is outlined in appendix B, starting from the approximate expressions given by Ohshima [23], under some conditions like $\kappa r_{0}>>1$ and parameters in the range of values used in this work, we can reach the same functional expression $V_{\mathrm{D}}=V_{\mathrm{D}}(I, Q)$ given by (15) for polyions modelled with spherical or cylindrical geometries.

This coincidence is an interesting fact since it means that Eqn. (15) can be used to reproduce results under planar, cylindrical and spherical geometries with a free (i.e. fitted) parameter $a$. This fitting procedure can compensate for the restrictions used in the derivation of Eqn. (15) as well as in the identification of $\psi_{\mathrm{S}}$ with $\psi_{\mathrm{D}}$.

\section{Materials and Methods}

\subsection{Experimental procedures}

Polyacrylic (PAA) and humic acids (HA) (H1, 675-2) were purchased from Aldrich. According to the manufacturer, this PAA had an average $\mathrm{M}_{\mathrm{w}}$ of 250000 . Stock solutions of $0.1 \mathrm{M}$ (in monomers) were prepared by dilution without further purification. Conversely, the HA was purified with the procedure outlined elsewhere [24] (molecules with molecular weight $<12000$ are discarded).

$\mathrm{KNO}_{3}$ Merck analytical grade was used as supporting electrolyte and $\mathrm{KOH}$ and $\mathrm{HNO}_{3} 0.1 \mathrm{M}$ Merck Titrisols as titrating solutions. Ultrapure water (Milli-Q plus 185 System, Millipore) was 
employed in all experiments. Purified water-saturated nitrogen was used for deaeration and blanketing of solutions.

To study the polyelectrolytic effect of PAA and HA, potentiometric acid-base titrations were performed at different ionic strengths. Potentiometric measurements were carried out with an Orion Research Ionanalyzer 720A attached to a Metrohm 665 Dosimat, for the automatic addition of solutions, and to a computer (with a data acquisition program) through a RS232 card. The potentiometric glass vessel was thermostated at $T=25.0^{\circ} \mathrm{C}$ and maintained under $\mathrm{N}_{2}$ pressure. The cell outlet was connected to a saturated $\mathrm{Ba}(\mathrm{OH})_{2}$ solution in order to prevent $\mathrm{CO}_{2}$ entry. pH was measured with a glass electrode Orion 9103.

In all experiments, a PAA or HA solution was placed into the potentiometric cell at a given concentration of $\mathrm{KNO}_{3}$ and was extensively deaerated and allowed to equilibrate. In order to avoid hysteresis effects the HA suspensions were first titrated to $\mathrm{pH} 7.0$ to ensure full dispersion and then back to $\mathrm{pH} 3.5$ [20] and they were then titrated to about $\mathrm{pH}$ 10.0. After each titrant addition, the reading was accepted when the rate of drift was less than $0.1 \mathrm{mV} \min ^{-1}$. A maximum reading time of 6 minutes was set for two consecutive readings of titrant, which was usually enough to obtain stable measurements.

As we need the bulk proton concentration to calculate the proton concentration at the macromolecular surface, the electrode system was directly calibrated to measure $\mathrm{H}^{+}$ concentration following Gran's method [25], in which a solution containing only the same salt level and some amount of a standard acid is titrated with the same standard base solution as in the sample titration. Due to this calibration method, hereafter we label

$$
\mathrm{pH}=-\log c_{\mathrm{H}}
$$

where $c_{\mathrm{H}}$ is the bulk proton concentration.

Two different kinds of experiments were performed:

a) Proton titrations of $50 \mathrm{~mL}$ of PAA $5 \cdot 10^{-3} \mathrm{M}$ in monomer at different ionic strengths, $c_{\mathrm{KNO}_{3}}=0.1,0.05,0.02,0.01$ and $0.005 \mathrm{M}$. 
b) Proton titrations of $25 \mathrm{~mL}$ of $\mathrm{HA} 0.5 \mathrm{~g} \mathrm{~L}^{-1}$ solution at different ionic strengths, $c_{\mathrm{KNO}_{3}}=0.05,0.01$ and $0.002 \mathrm{M}$.

The titration curves obtained under the different experimental conditions studied were transformed into $Q$-pH curves, with the charge $Q$ of the polyelectrolyte in mol $\mathrm{L}^{-1}$ for PAA and in $\mathrm{mol} \mathrm{kg}^{-1}$ for HA. For Humic acids, $Q$ can be calculated through the balance:

$$
Q=Q^{0}+\Delta Q=Q^{0}+\left(c_{\mathrm{B}}-\left(\frac{V}{V+v}\left(c_{\mathrm{H}}^{0}-c_{\mathrm{OH}}^{0}\right)-c_{\mathrm{H}}+c_{\mathrm{OH}}\right)\right) \frac{1}{c_{\mathrm{P}}}
$$

where $Q^{0}$ is the initial charge (before any base addition, determined from the initial $\mathrm{pH}$ ), $\Delta Q$ is the increment of charge in each point, $V$ is the initial volume and $v$ the total added volume, $c_{\mathrm{B}}$ is the concentration in the system of base added, $c_{\mathrm{H}}$ and $c_{\mathrm{OH}}$, the concentrations of $\mathrm{H}^{+}$and $\mathrm{OH}^{-}$, respectively, all in mol $\mathrm{L}^{-1}$ and $c_{\mathrm{P}}$ is the concentration of $\mathrm{HA}$ in $\mathrm{kg} \mathrm{L}^{-1}$. The superscript ${ }^{0}$ refers to the initial solution. For PAA the charge $Q$ will be expressed in $\mathrm{mol} \mathrm{L}^{-1}$, so that, for this system one can use eqn. (17) without the last factor (i.e. without dividing by $c_{\mathrm{P}}$ ).

\subsection{Fitting procedure}

One fitting strategy used in the literature [26,27] is the merging of $Q v s$. $\mathrm{pH}$ at different ionic strengths into a unique "master curve", $Q$ vs. $\mathrm{pH}_{\mathrm{s}}$, (recall $\left.\mathrm{pH}_{\mathrm{s}}=-\log c_{\mathrm{H}_{\mathrm{s}}}\right)$. Then in a second step, parameters corresponding to the specific binding are obtained from the fitting of the master curve. Another fitting strategy is based on the minimization of the differences between the raw experimental measurements along the titrations and the corresponding calculated values. Among the various possible measurements (coverage, $\log ($ coverage), charge, etc.) we have chosen the fitting of the experimental $\mathrm{p} K_{\text {app }} v s . \mathrm{pH}$ (or vice-versa) to the predicted ones. This strategy requires the simultaneous assumption of both, a polyelectrolyte correction methodology for the electrostatic binding, and a complexation isotherm for the specific one in order to obtain the calculated $\mathrm{p} K_{\text {app }}$ values. In this way, parameters embedded in the Donnan volume are fitted 
simultaneously to the parameters of the isotherm describing the specific binding. The merging of all the titration curves into one master curve, not imposed in this alternative strategy, can now be seen as a check of the goodness of the method.

\section{A testing system: Proton binding to PAA}

Proton binding to PAA has been largely studied in the literature that developed the classical methods to deal with the electrostatic binding. Here, this system will be used as a model for linear and homogeneous polyelectrolytes, in order to check the Donnan methodology with the new expression for $V_{\mathrm{D}}$.

Fig. 1 shows the experimental concentration of deprotonated groups of PAA as a function of $\mathrm{pH}$ at different ionic strengths. Notice that at any fixed $\mathrm{pH}$, higher charges correspond to larger $I$, since an increase in the screening of the macromolecular charge by the ionic atmosphere facilitates the dissociation of protons.

\subsection{Reference approach: cylindrical Poisson-Boltzmann}

Experimental $Q$ vs. $\mathrm{pH}$ curves can be converted into $Q v s$. $\mathrm{pH}_{\mathrm{S}}$ by applying (A.3) after the numerical solving of the Poisson-Boltzmann model (to obtain the surface potential $\psi_{\mathrm{S}}$ ) assuming cylindrical geometry with radius of the cylinder $r=0.55 \mathrm{~nm}[28]$ and structural charge density $\xi_{\text {str }}^{\text {cyl }}=2.85[28,29]$. As can be seen in Fig. 1, the curves at different ionic strength collapse quite well into a master curve, $Q$ vs. $\mathrm{pH}_{\mathrm{S}}$, (labelled NLPB in the figure) suggesting a successful correction of the electrostatic effects within the mean field approximation.

In Fig. 2, together with $\mathrm{p} K_{\text {app }}$ of the PAA titrations of Fig. 1, we depict the corresponding $\log K_{\mathrm{c}}$ values, obtained from Eqs. (1) and (2) after the polyelectrolytic correction. We highlight the direct physical meaning of this plot, since $K_{\mathrm{c}}$ gives at each proton concentration the mean value of the specific binding constants to the remaining deprotonated sites [15]. While a continuous increase in $\mathrm{p} K_{\text {app }}$ is observed as $\alpha_{\mathrm{d}}$ or $\mathrm{pH}$ increase, $\log K_{\mathrm{c}}$ is almost independent of the ionic strength as expected once the electrostatic effects have been corrected and it shows a weak 
dependence on the $\mathrm{pH}$ or dissociation degree of the macromolecule. The approximate constant $\log K_{\mathrm{c}}$-value indicates that all the proton binding sites show a common affinity (a common value of the binding constant to the specific site) so that proton binding can be mainly explained with a homogeneous and independent complexation model. The mild dependency of $\log K_{\mathrm{c}}$ on $\mathrm{pH}$ could be the result of experimental errors, or a remaining interaction energy between sites, for instance from electrostatic origin due to limitations of the mean field approximation in the description of the electrostatic binding at high charge values of the polyelectrolyte.

Even though the specific stability constant of ideal ligands (i.e. ligands exhibiting homogeneous and independent complexation) can be obtained by extrapolation of $K_{\mathrm{c}}$ at $\alpha_{\mathrm{d}} \rightarrow 0$ [28], we will now proceed in a more general way in order to quantify the deviations from ideal complexation $[14,30]$. This way consists in fitting the $\log K_{\mathrm{c}} v s . \mathrm{pH}_{\mathrm{S}}$-couples of the master curve to the predicted values obtained by means of the different isotherms reported in Table 1 . The values of the standard deviation, $\sigma$ (eqn. (6)), of the affinity spectrum associated to each isotherm indicate the heterogeneous or homogeneous character of the binding, i. e., the $\sigma$-values are a measure of the thickness of this distribution. If the affinity of all the sites for proton is the same, the affinity spectrum tends to a Dirac delta function and the standard deviation, $\sigma$, tends to zero. Table 2 shows the parameters obtained in the fitting of each isotherm and the mean $(\mu)$ and the standard deviation $(\sigma)$ of the corresponding affinity spectra. All three isotherms yield very close binding results when they are compared in terms of $\mu$ and $\sigma$ of the affinity spectra. The average of these $\mu$ values, $\mu=\left(\log k_{\mathrm{m}} / \mathrm{M}^{-1}\right)=(4.41 \pm 0.01)$, is not far from the affinity of the proton for the acetate group and in agreement with values reported in the literature [28]. The average standard deviation obtained, $\sigma=\left(\sqrt{\left\langle\log ^{2}\left(k_{\mathrm{m}} / \mathrm{M}^{-1}\right)\right\rangle-\mu^{2}}\right)=(0.48 \pm 0.03)$, quantifies the deviations from homogeneous binding as explained above. 


\subsection{Donnan approach with the new $V_{\mathrm{D}}$ expression}

We have simultaneously fitted the parameters of the different isotherms and the parameters of $V_{\mathrm{D}}$ given by (15) by minimizing the corresponding $\chi^{2}$ obtained by adding up the squares of the differences between the experimental and predicted $\mathrm{p} K_{\text {app }} v s$. $\mathrm{pH}$-couples (or vice-versa). Table 3 summarizes the values obtained for the different parameters and the corresponding value for the mean and standard deviation of the affinity spectrum associated to each isotherm. Notice that the three isotherms considered yield similar mean affinity values, average of these values $\mu=(4.41 \pm 0.04)$, average variance $\sigma=(0.49 \pm 0.04)$ and Donnan parameter $a=(190 \pm 15) \mathrm{M}^{-}$

${ }^{1 / 2}$. Both, the values of the mean affinity and of variance are very close to those obtained using NLPB in cylindrical geometry (see section 5.1). With the average value of $a$, plots of $Q v s \mathrm{pH}_{\mathrm{S}}$ and $\log K_{\mathrm{c}} v s \mathrm{pH}_{\mathrm{S}}$ have been depicted in Figures 1 and 2, respectively. A collapse of the different titrations into a master curve (labelled D) is seen indicating a good description of the electrostatic binding with results almost coincident with those coming from the NLPB description. The constancy of $a$ in Table 3 for the different isotherms indicates the independence of the Donnan correction on the isotherm considered.

\section{Proton binding to HA}

Humic acids are operationally defined as complex mixtures with two major functional groups, carboxylic and phenolic. Their association constants appear to lie within the range $\log K_{\mathrm{H} 1}=$ $[1.99,3.90]$ and $\log K_{\mathrm{H} 2}=[6.06,10.06]$, respectively (values reported by Milne et al. from the fitting of the NICCA-Donnan model to 49 data sets [31]).

Fig. 3 shows the titration curves of purified Aldrich humic acid at different ionic strengths. In the plot, there seems to be two wide inflection points, confirming the bifunctional character of this humic acid. Although a certain plateau can be observed between both inflection points, the determination of the $\mathrm{pH}$ at which a specified group is neutralized can be somewhat arbitrary.

It has been common practice to rely upon some simple, empirical rules to estimate the concentration of carboxyl and phenolic groups. For example, it is often assumed that all carboxyl 
groups (but no phenolic group) are titrated by $\mathrm{pH} 7.0$ [32,33] or $\mathrm{pH} 8.0$ [32-35]. Graphical methods have been designed by establishing end points for $\mathrm{COOH}$ through first- or secondderivative plots: the maximum of the $\mathrm{dpH} / \mathrm{d} v v s$. titrant volume $v$ or the volume $v$ where $\mathrm{d}^{2} \mathrm{pH} / \mathrm{d} v^{2}$ $=0$. The volume below this point can then be taken to be associated with carboxyl groups and the volume above this point as due to phenolic groups and other weak acids. Following this procedure, a $\mathrm{pH}$ limit of 8 has been found for our HA from experimental data. In this range (a $\mathrm{pH}$ window of $[3.5,8]$ ), we assume that we can describe the affinity seen by the proton through a unimodal distribution function. The maximum charge corresponding to this $\mathrm{pH}$ range has been estimated to be $Q_{\max }=(3.56 \pm 0.22) \mathrm{mol} \mathrm{kg}^{-1}$ and this value is used here when specific coverages are required.

\subsection{Reference approach: spherical Poisson-Boltzmann}

Humic and fulvic acids have been represented as rigid spheres [27,36,37] or rigid cylinders [27,34]. The rigid sphere model represents compact humics, whereas the cylindrical model represents worm-like humics. Some recent publications support the assumption of a spherical geometry $[21,38,39]$, and consequently only this geometry will be considered here when solving the Poisson Boltzmann equation for the humic acid.

The protonation curves at different ionic strengths for the humic acid in the $\mathrm{pH}$-range [3.5-8] are shown in Fig. 4 and, as reported elsewhere [21],[37] for a fixed pH, the charge increases with increasing salt concentration, as expected.

The solution of the Poisson-Boltzmann equation assuming spherical geometry transforms our experimental data into a protonation master curve (see curve labelled NLPB in Figure 4). Two parameters are required for this process, the sphere radius, $r_{0}$, and the maximum charge of the macromolecule that we express in terms of the number of surface charged groups per molecule, $n_{\mathrm{p}}$. As these values are unknown, both parameters $r_{0}$ and $n_{\mathrm{p}}$ can be fitted simultaneously, by solving the Poisson-Boltzmann equation and requiring that the $\log K_{\mathrm{c}}$-curves at different ionic 
strengths merge into a single master curve (see Fig. 4 and 5). The fitted $r_{0}=3.5 \mathrm{~nm}$ and $n_{\mathrm{p}}=30$ values lie within the ranges reported in the literature [27].

Following the minimization procedure outlined in section 4.2 , the electrostatic correction of $\mathrm{p} K_{\text {app }}$ yields $\log K_{\mathrm{c}}$ values clearly dependent on $\alpha_{\mathrm{d}}$ (Fig. 5). This dependence supports the heterogeneous character of the proton binding to HA and agrees with previous studies [40] that report a variety of types of carboxylic acids in humic acids, each of which is likely to have its own intrinsic affinity. Moreover, very similar values of $\mu$ and $\sigma$ of the three complexation isotherms considered are obtained (Table 4), their averages being $\mu=(4.18 \pm 0.01)$, and $\sigma=(1.7 \pm 0.1)$. Notice that the $\mu$-value obtained is not far away from that obtained for the PAA since a common functional group is involved in the sites considered for both systems. However, the $\sigma$-value for the $\mathrm{HA}$ is much higher than that of the PAA reflecting the heterogeneous character of the HA.

\subsection{Existing approach: Donnan model with $V_{\mathrm{D}}(I)$}

It has been reported that the Donnan approach can be successfully applied to humics if the specific volume of the humic substances is dependent on the ionic strength; but not necessarily on the charge [13]. An empirical relationship between the volume of the Donnan phase and the ionic strength is given by eqn. (11). Using this $V_{\mathrm{D}}$ expression with $b=0.43$ (this value is found in the literature as reasonable for humic acids [13]), $Q$ vs $\mathrm{pH}_{\mathrm{S}}$ and $\log K_{\mathrm{c}} v s \mathrm{pH}_{\mathrm{S}}$ curves merge into a unique master curve labelled $\mathrm{D}(11)$ (Figs. 4 and 5, respectively). The free adjusting of $b$ by minimizing the experimental $\mathrm{p} K_{\text {app }} v s$. $\mathrm{pH}$-couples does not introduce relevant changes. The dependency of $\log K_{\mathrm{c}}$ on the charge seen in Fig. 5 is consistent with the heterogeneous character of the HA and can be interpreted with different heterogeneous isotherms (Frumkin, LangmuirFreundlich and uniform). Table 5 summarizes the binding parameters fitted for each isotherm. In terms of the affinity distributions underlying the three different isotherms, the values of $\mu$ and $\sigma$ of each affinity distribution are very similar, with average $\mu=(2.93 \pm 0.02)$, and $\sigma=(1.8 \pm 0.3)$. 
In comparison with the values obtained with the NLPB treatment (see Table 4), a difference of around one unit in the mean specific affinity is found, while both treatments yield similar variances (slightly lower in the NLPB treatment). Thus, the affinity distribution is interpreted as having similar heterogeneity with the mean of the distribution shifted one $\log K_{\mathrm{c}}$ unit [37] between both treatments, the electrostatic binding being overestimated by the Donnan treatment (with respect to NLPB).

Although a good convergence of the different titrations in one master curve is obtained in this implementation of the Donnan model, two criticisms arise when analyzing $D(11)$ : i) the associated Boltzmann factor, $\exp \left(-\tilde{\psi}_{\mathrm{D}}\right)$, exhibits a very reduced dependence on $Q$. Notice that the Boltmann factor is just given by the difference in the abscissa values of a titration curve and the master curve $\mathrm{D}(11)$ at a fixed ordinate value. Although this difference is expected to increase when the macromolecular charge increases, this increase is very small in the present implementation of the Donnan model as can be seen in Fig. 4.; ii) A second criticism is that the polyelectrolytic correction at low $\alpha_{\mathrm{d}}$-values seen in Fig. 4 is larger than expected (when $\alpha_{\mathrm{d}}$ tends to zero a unity Boltzmann factor is expected for a finite value of $V_{\mathrm{D}}$ ). This behaviour is not specific of the present HA since high values of the Boltzmann factor for low $\alpha_{\mathrm{d}}$ have also been reported in the literature [13] in the application of the Donnan model to HA of other origins.

\subsection{Donnan approach with the new $V_{\mathrm{D}}$ expression}

Parallel to the procedure described in section 5.2, the Donnan treatment is applied in this section to the HA data with $V_{\mathrm{D}}$ given by (15). Among the advantages of this expression we highlight its direct connection with the NLPB treatment for different geometries and the explicit dependence of $V_{\mathrm{D}}$ on the charge of the macromolecular polyion. Fitted parameters are gathered in table 6 , with average values $\mu=(4.34 \pm 0.04), \sigma=(1.8 \pm 0.2)$ and $a=0.030 \pm 0.002 \mathrm{~kg}(\mathrm{~mol} . \mathrm{L})^{-1 / 2}$. Although not imposed by the applied fitting procedure, all titration curves merge quite well into a single master curve labelled $\mathrm{D}(15)$ in Figs. 4 and 5. This master curve, $\mathrm{D}(15)$, diverges from the previous implementation of the Donnan model, $\mathrm{D}(11)$, while it approaches the NLPB results. 
This is not surprising since $V_{\mathrm{D}}$ given by (15) is an approximation for NLPB under different geometries.

\subsection{Comparison of the approaches}

As already commented, different methods applied to consider the electrostatic binding yield collapses into quite different master curves. This behaviour is also found when both the electrostatic and the specific binding are fitted simultaneously, as done in the present application of the Donnan model, and also by using different isotherms to describe the specific binding. In this way, the agreement of the Donnan volume fitted parameters and of $\mu$ and $\sigma$ for the different isotherms assayed, although being a criterion that supports the reliability of the results obtained, could still be not enough to determine a unique master curve. Thus, the present results suggest caution in the comparison of the binding parameters obtained with different methods.

Expression (15), here introduced for the Donnan volume, while extremely simple in terms of numerical manipulation, gives results close to NLPB which can be considered as a reference method within the mean field approximation. With the present data, the differences between the values predicted by D (with $V_{\mathrm{D}}$ given by (15)) and NLPB are around 7\%.

Moreover, the almost parallel behaviour of the master curve $\mathrm{D}(15)$ to the titration curves suggests that -at least within the approximation given by (15)- the charge does not play a key role in the polyelectrolytic correction of the studied HA, probably because of the low volumic charge densities involved, supporting previous assumptions in the literature of $V_{\mathrm{D}}=V_{\mathrm{D}}(I)$ as prescribed by eqn. (11). Actually, in the limit of low charge $\left(a\left(\frac{(\mathrm{mol} \mathrm{L})^{1 / 2}}{\mathrm{~kg}}\right) \ll 1\right), V_{\mathrm{D}}$ given by (15) becomes similar to (11), $V_{D} \propto \frac{1}{4 a} I^{-0.5}$, being the exponent of the ionic strength comparable to the one reported previously $(0.43$ in ref. [13]) but with a different preexponential factor $(1 / 4 a$ instead of $\left.10^{(b-1)}\right)$. This indicates that expression (15) allows to justify the empirical dependence 
observed $V_{\mathrm{D}}=V_{\mathrm{D}}(I)$ and suggests that the expression for the Donnan volume, given in (11), which only takes into account the dependence on ionic strength, can be generalized, by including two adjustable parameters, to

$V_{\mathrm{D}}=c I^{-b}$

Using expression (18) coupled to the isotherms, the fitting of the titration data yields a master curve almost indistinguishable from $\mathrm{D}(15)$. Thus the values of the isotherm parameters become quite close to those obtained with (15) (reported in table 6), $b$ becomes $b=0.40 \pm 0.03$, close to the fixed $b$-value used in (11) and $c=(10 \pm 3) \mathrm{kg}^{-1}(\mathrm{~mol} \mathrm{~L})^{1 / 2}$, similar to the preexponential value obtained from (15), $\frac{1}{4 a} \approx 9 \mathrm{~kg}^{-1}(\mathrm{~mol} \mathrm{~L})^{1 / 2}$.

Finally, we notice that the HA appears to be more heterogeneous by means of the Donnan description than with the NLPB treatment, while the Donnan treatment with expression (15) seems to underestimate the surface potential with respect to NLPB.

A last remark concerns the freedom of choice of different isotherms in the description of the specific binding. As reported in Tables 4-6, the mean and variance of the affinity distributions underlying the different isotherms used in the present work converge, this indicating the robustness of these values to characterise the specific binding and the freedom in the choice of the isotherm. This result, here observed with the proton-HA binding, agrees with previous theoretical predictions [15].

\section{Concluding remarks}

A new approximate analytical expression for the Donnan volume, given by eq. (15), has been introduced by combining the Donnan basis for the description of the electrostatic binding with the results of the classical non linear Poisson-Boltzmann equation. This $V_{\mathrm{D}}$ expression depends on the ionic strength and on the degree of dissociation with only one adjustable parameter. It is 
independent of the kind of polyelectrolyte, homogeneous or heterogeneous and able to describe different geometries of the polyelectrolyte.

The Donnan methodology with this new $V_{\mathrm{D}}$ expression has been applied to PAA (as an example of homogeneous polyelectrolyte) and to HA (as an example of heterogeneous one) together with NLPB, used as a reference, and previous implementations of the Donnan model found in the literature. The master curves resulting from the various polyelectrolytic treatments do not necessarily coincide indicating that the splitting of the electrostatic and intrinsic contributions to the binding energy is not unique, even when the electrostatic and specific binding are fitted simultaneously.

Results obtained in the Donnan treatment using eq. (15) while benefiting from a extremely simple numerical manipulation, are close to the results of the NLPB treatment in each case. Moreover, Equation (15) allows to justify empirical expressions for the Donnan volume found in the literature. These findings support the use of this new expression for the Donnan volume, depending on ionic strength and charge, for the description of the electrostatic binding to a general macromolecular ligand.

The specific binding has been described by using different adsorption isotherms (LangmuirFreundlich, Frumkin and uniform). Consistency of the results of the specific binding among the isotherms has been seen by computing the average $\mu=\langle\log k\rangle$ and the variance $\sigma^{2}=\left\langle(\log k)^{2}\right\rangle-\mu^{2}$ of the affinity spectrum underlying each isotherm. Similar $\mu$ and $\sigma$ values are obtained in each case.

The low $\sigma$-values obtained in the PAA case confirm that the proton binding to this polyion is mainly homogeneous and independent. The heterogeneity of the proton binding to the humic acid has been quantified by the variance of the affinity spectrum underlying the different isotherms. 


\section{Acknowledgements}

The authors gratefully acknowledge support of this research by the Spanish Ministry of

Education and Science (DGICYT: Projects BQU2003-09698 and BQU2003-07587), by the European Community under contract EVK1-CT2001-86, from the "Comissionat d'Universitats i Recerca de la Generalitat de Catalunya".

\section{Appendix A: Solution of the NLPB equation}

NLPB equation for the electrostatic potential, $\psi$, created by a polyelectrolyte in presence of only one symmetrical $z: z$ supporting electrolyte, which screens the charges of the polyelectrolyte, is

$$
\nabla^{2} \psi=-\frac{\rho}{\varepsilon}=\kappa^{2} \frac{k_{\mathrm{B}} T}{z e} \sinh (z \psi)
$$

where $\kappa$ is the reciprocal Debye's length

$$
\kappa=\left(\frac{e^{2} N_{\mathrm{A}} 2 I}{\varepsilon k_{\mathrm{B}} T}\right)^{1 / 2}
$$

$N_{\mathrm{A}}$ is Avogadro number, $\varepsilon$ is the bulk dielectric constant and $k_{\mathrm{B}}$ is Boltzmann constant.

The solution of equation (A.1) depends on the concentration of the supporting electrolyte through $I$, on the geometry of the polyelectrolyte, which is considered in this model as a rigid and impermeable surface, and on the charge of the polyelectrolyte through the boundary condition at the surface. The remaining boundary condition prescribes that the electrostatic potential vanishes at infinite (far away from any molecule of polyelectrolyte in dilute situations).

Notice that the Boltzmann relationship is embedded in (A.1) and, when applied to the proton, states

$$
c_{\mathrm{H}_{\mathrm{S}}}=c_{\mathrm{H}} \exp \left[\frac{-e \psi_{\mathrm{S}}}{k_{\mathrm{B}} T}\right]
$$


where $c_{\mathrm{H}_{\mathrm{S}}}$ labels the proton concentration at the macromolecular surface. Expression (A.3) is a very simple case of (8). We recall that $\psi_{\mathrm{S}}$, the electric potential at the macromolecular surface, is due to all the charges present in the system, including ions and the polyion. However, $\psi_{\mathrm{S}}$ obtained from (A.1) (within NLPB) is only an approximate value of this potential. Notice, for instance, that NLPB does not introduce any correction for the ionic interaction in the bulk of the solution. Beyond the mean field approximation, the concentration of counterions at the surface is found to be even higher than predicted by (A.3) due to correlation effects [41].

In order to simplify the notation it is convenient to introduce dimensionless quantities. Defining

$$
\tilde{\psi} \equiv \frac{e \psi}{k_{\mathrm{B}} T}
$$

and assuming spherical or cylindrical geometry, the NLPB equation becomes an ordinary differential equation (ODE) of second order, which in a compact form can be written as

$$
\frac{d^{2} \tilde{\psi}}{d r^{2}}+\frac{g}{r} \frac{d \tilde{\psi}}{d r}=\frac{\kappa^{2}}{z} \sinh (z \tilde{\psi})
$$

where $g=1$ for cylindrical geometry and $g=2$ for spherical geometry.

The value boundary problem is:

$$
\psi(\infty)=0 ; \quad\left(\frac{d \psi}{d r}\right)_{r=r_{0}}=-\frac{\sigma}{\varepsilon}
$$

where $r_{0}$ represents the surface coordinate, i.e., either the radius of the cylinder or the radius of the sphere, respectively, and $\sigma$ is the surface charge density.

Taking dimensionless radial coordinates in logarithmic space

$$
\begin{aligned}
& y \equiv \kappa r ; \quad x=\ln y \\
& y_{0} \equiv \kappa r_{0} ; \quad x_{0}=\ln y_{0}
\end{aligned}
$$

the NLPB equation (A.5) becomes 


$$
\frac{d^{2} \tilde{\psi}}{d x^{2}}+(g-1) \frac{d \tilde{\psi}}{d x}=\frac{e^{2 x}}{z} \sinh (z \tilde{\psi})
$$

and the value problem boundary (A.6) becomes

$$
\tilde{\psi}(\infty)=0
$$

$\left(\frac{d \tilde{\psi}}{d x}\right)_{x=x_{0}}=-2 \beta$

for cylindrical geometry and

$$
\left(\frac{d \tilde{\psi}}{d x}\right)_{x=x_{0}}=-\beta
$$

for spherical geometry. $\beta$ is related to the dimensionless surface charge density. In cylindrical geometry, $\beta$ is defined as

$$
\beta=z_{\mathrm{p}} \xi_{\mathrm{str}}^{\mathrm{cyl}} \alpha_{\mathrm{d}}
$$

in terms of the structural charge density, $\xi_{\mathrm{str}}^{\mathrm{cyl}}=l_{\mathrm{B}} / b_{\mathrm{d}}$, where $l_{\mathrm{B}}$ is the Bjerrum length and $b_{\mathrm{d}}$ is the separation of charged ionised functional groups, and $z_{\mathrm{p}}$ is the charge of one functional group of the polylectrolyte. And, for spherical geometry

$\beta=z_{\mathrm{p}} \xi_{\mathrm{str}}^{\mathrm{sph}} n_{\mathrm{p}} \alpha_{\mathrm{d}}$

where $\xi_{\mathrm{str}}^{\mathrm{sph}}=l_{\mathrm{B}} / r_{0}$ and $n_{\mathrm{p}}$ is the maximum number of ionisable groups over the surface (in practice, of the whole polyelectrolyte).

In the present work, the numerical solution of the NLPB equation for cylindrical and spherical geometries are obtained through the Galerkin Finite Element Method [42].

\section{Use of the GFEM method}

For the sake of simplicity we detail here only the particular case of cylindrical geometry $(g=1)$. 
Using the GFEM method, the integral formulation of eq. (A.8) reads

$$
\int_{x_{0}}^{L} \omega_{k}(x)\left(\frac{e^{2 x}}{z} \sinh (z \tilde{\psi})-\frac{d^{2} \tilde{\psi}}{d x^{2}}\right) d x-\omega_{k}\left(x_{0}\right)\left\{\left(\frac{d \tilde{\psi}}{d x}\right)_{x=x_{0}}+2 \beta\right\}=0
$$

where $L$ is an arbitrary distance, sufficiently large so that the boundary condition at $x \rightarrow \infty$ is fulfilled at this position and $\left\{\omega_{k}(x)\right\}$ is a set of arbitrary functions of the space functions.

Then, integration by parts of the above integral equation yields the weak formulation of the NLPB ODE equation

$$
\int_{x_{0}}^{L} \omega_{k}(x) \frac{e^{2 x}}{z} \sinh (z \tilde{\psi})+\int_{x_{0}}^{L}\left(\frac{d \omega_{k}(x)}{d x}\right)\left(\frac{d \tilde{\psi}}{d x}\right) d x-\omega_{k}\left(x_{0}\right) 2 \beta=0
$$

Now, the unknown function, $\tilde{\psi}(x)$, is expanded as a function of linear spatial interpolants with two sets of unknown coefficients $m_{j}$ and $u_{j}$

$$
\tilde{\psi}(x)=\frac{1}{z} \sum_{j=0}^{N-1} m_{j} \omega_{j}(x) ; \sinh \{z \tilde{\psi}(x)\}=\sum_{j=0}^{N-1} u_{j} \omega_{j}(x)
$$

The interpolants are linear piecewise functions (see Figure 6) defined as

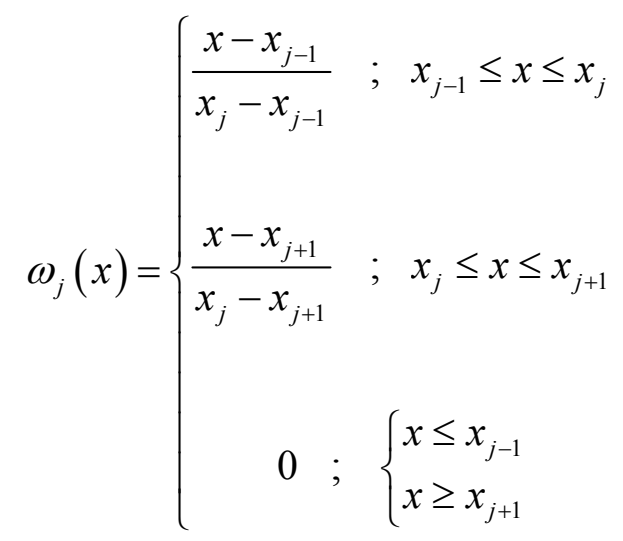

In order to relate the two unknown set of coefficients, we notice that at a point grid $j$, all the interpolants (except $\omega_{j}$ which becomes 1) vanish, so the value of the function $\tilde{\psi}(x)$ takes the value $m_{j}$ at $x=x_{j}$. Similarly, the function $\sinh \tilde{\psi}(x)$ takes the value $u_{j}$ at $x=x_{j}$; therefore we conclude $u_{j}=\sinh m_{j}$. 
Then, the terms of the above integral equation can be computed analytically and it reduces to a system of $N$ non-linear equations which can be written in a matrix form as

$$
\mathbf{A u}+\mathbf{C m}+\mathrm{w}=\mathbf{0}
$$

where

$$
\mathrm{u}=\left(\begin{array}{c}
u_{0} \\
u_{1} \\
\vdots \\
u_{N-1}
\end{array}\right)=\left(\begin{array}{c}
\sinh m_{0} \\
\sinh m_{1} \\
\vdots \\
\sinh m_{N-1}
\end{array}\right) ; \mathrm{m}=\left(\begin{array}{c}
m_{0} \\
m_{1} \\
\vdots \\
m_{N-1}
\end{array}\right) ; \mathrm{w}=\left(\begin{array}{c}
-2 \beta \\
0 \\
\vdots \\
0
\end{array}\right)
$$

and the matrices are

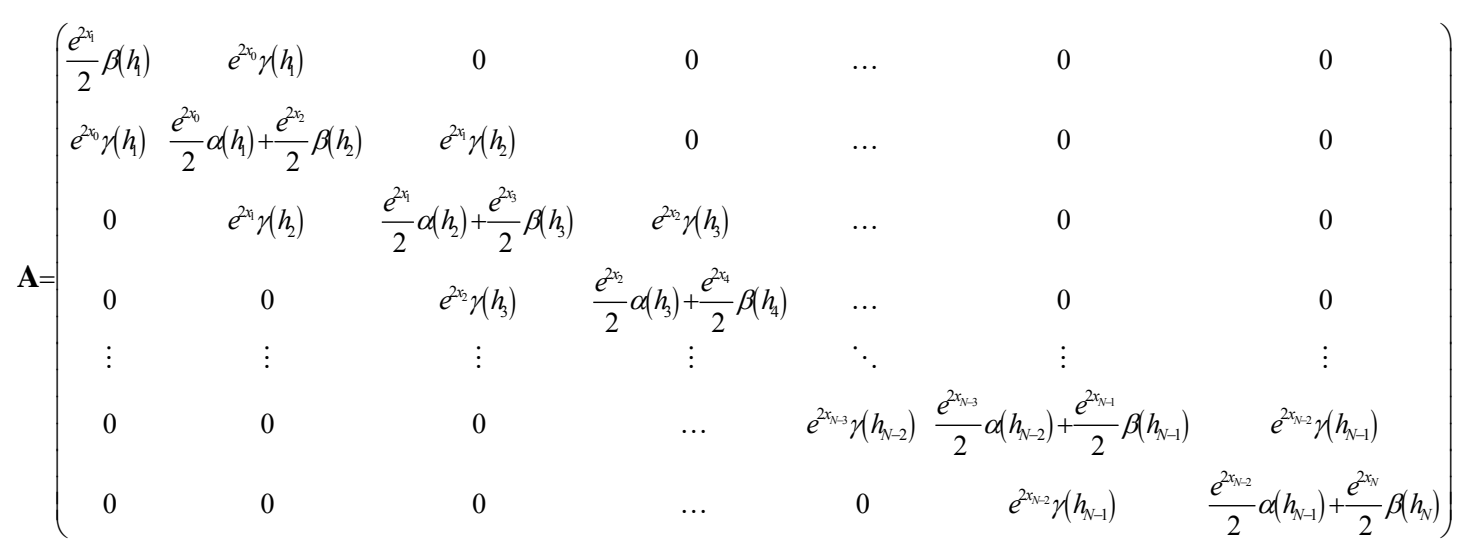

where

$$
\begin{aligned}
& \alpha(h) \equiv \frac{1}{2 h^{2}}\left(e^{2 h}-1\right)+e^{2 h}\left(1-\frac{1}{h}\right) \\
& \beta(h) \equiv \frac{1}{2 h^{2}}\left(1-e^{-2 h}\right)-e^{-2 h}\left(1+\frac{1}{h}\right) \\
& \gamma(h) \equiv \frac{1}{4 h}\left\{e^{2 h}\left(1-\frac{1}{h}\right)+\left(1+\frac{1}{h}\right)\right\}
\end{aligned}
$$

and 


$$
\mathbf{C}=\left(\begin{array}{cccccc}
\frac{1}{h_{1}} & -\frac{1}{h_{1}} & 0 & \cdots & 0 & 0 \\
-\frac{1}{h_{1}} & \frac{1}{h_{1}}+\frac{1}{h_{2}} & -\frac{1}{h_{2}} & \cdots & 0 & 0 \\
0 & -\frac{1}{h_{2}} & \frac{1}{h_{2}}+\frac{1}{h_{3}} & \cdots & 0 & 0 \\
\vdots & \vdots & \vdots & \ddots & \vdots & \vdots \\
0 & 0 & 0 & \cdots & \frac{1}{h_{N-2}}+\frac{1}{h_{N-1}} & -\frac{1}{h_{N-1}} \\
0 & 0 & 0 & \cdots & -\frac{1}{h_{N-1}} & \frac{1}{h_{N-1}}+\frac{1}{h_{N}}
\end{array}\right)
$$

with $h_{j}=x_{j+1}-x_{j}$, representing the distance between the points in the grid, which in general can vary adapting to the requirements of the particular problem at different positions. Here, due to the fact that we have chosen the radial distance in logarithmic space, there is no need to use an uneven grid, so $h_{j}=h$ has been used. We have used 200 points in the grid with $L$ sufficiently large.

This system of non-linear equations is solved numerically using a Newton-Raphson method [43].

\section{Appendix B: Approximate expression for the Donnan volume}

\section{Cylindrical geometry}

In order to obtain a simplified expression for $V_{\mathrm{D}}$ using eqn. (12), one can estimate the surface potential from the approximate analytical solution of the NLPB equation for cylindrical geometry given by Ohshima [23]:

$\sigma=\frac{\sqrt{8 \varepsilon R T I}}{z} \sinh \left(\frac{z \tilde{\psi}_{\mathrm{S}}}{2}\right)\left[1+\frac{\beta_{0}^{-2}-1}{\cosh ^{2}\left(z \tilde{\psi}_{\mathrm{S}} / 4\right)}\right]^{1 / 2}$

with 


$$
\beta_{0}=\frac{K_{0}\left(\kappa r_{0}\right)}{K_{1}\left(\kappa r_{0}\right)}
$$

where $K_{\mathrm{n}}(z)$ is the nth order modified Bessel function of the second kind.

We suggest two different approximations, labelled A and B:

A) In the first approximation, given the range of values of $\kappa$ and $r_{0}$ appearing in this work, typical of natural humic matter, we use two further simplifications in eqn. (B.1):

$$
\left(1+\frac{\beta_{0}^{-2}-1}{\cosh ^{2}\left(z \tilde{\psi}_{\mathrm{S}} / 4\right)}\right)^{1 / 2} \approx \frac{\beta_{0}^{-1}}{\cosh \left(z \tilde{\psi}_{\mathrm{S}} / 4\right)}
$$

and

$$
\beta_{0}=\frac{K_{0}\left(\kappa r_{0}\right)}{K_{1}\left(\kappa r_{0}\right)} \approx \frac{\kappa r_{0}}{c+\kappa r_{0}}
$$

with a suitable constant $c$. Writing $\sinh z \tilde{\psi}_{\mathrm{S}}=\sinh z \tilde{\psi}_{\mathrm{D}}$ of eqn. (B.1) in terms of $\sinh \left(z \tilde{\psi}_{\mathrm{S}} / 4\right)$, which can be isolated from (B.1) with the aforementioned approximations, we derive

$$
V_{\mathrm{D}} \simeq \frac{Q z}{2 I\left[8\left(\frac{a Q z}{b+\sqrt{I}}\right)^{3}+4\left(\frac{a Q z}{b+\sqrt{I}}\right)\right] \sqrt{1+\left(\frac{a Q z}{b+\sqrt{I}}\right)^{2}}}
$$

where $a$ and $b$ are constants (gathering other constants) which are left free for the fitting to the considered experiments. The application of expression (B.5) to our data yield very similar results to the simpler approximation given by (15) (which only involves one adjustable parameter), so no further comment is included in this work.

B) In the second, and rougher, approximation, we just take the term in between square brackets in (B.1) as a free constant. Expressing $\sinh z \tilde{\psi}_{\mathrm{S}}=\sinh z \tilde{\psi}_{\mathrm{D}}$ of eqn. (12) in terms of $\sinh \left(z \tilde{\psi}_{\mathrm{S}} / 2\right)$, 
like we have done for planar geometry, we finally obtain eqn. $V_{D} \simeq \frac{1}{4 a \sqrt{I+a^{2} Q^{2} z^{2}}}$, which is (15).

\section{Spherical geometry}

The starting approximate NLPB eqn for spherical geometry is [23]

$$
\sigma=\frac{\sqrt{8 \varepsilon R T I}}{z}\left[2 \sinh \left(\frac{z \tilde{\psi}_{\mathrm{S}}}{2}\right)+\frac{4}{\kappa r_{0}} \tanh \left(\frac{z \tilde{\psi}_{\mathrm{S}}}{4}\right)\right]
$$

With the parameters used in this work, the tanh term can be neglected, and parallel to approximation B in the cylindrical approximation above explained, we obtain again (15). In this way, a Donnan volume expression, independent of the geometry (planar, cylindrical or spherical) is suggested. 


\section{References}

[1] J.Buffle, Complexation Reactions in Aquatic Systems: An Analytical Approach, Ellis Horwood Ltd ed. Chichester, 1988.

[2] C.F.Anderson, M.T.Record, Annu. Rev. Biophys. Chem. (1990) 423.

[3] K.A.Sharp, B.Honig, Annu. Rev. Biophys. Chem. 19 (1990) 301.

[4] F.Oosawa, N.Imai, I.Kagawa, J. Polym. Sci. 13 (1954) 93.

[5] A.Katchalsky, Z.Alexandrowicz, O.Kedem, in B. E. Conway and R. G. Barradas (Eds.), Chemical physics of ionic solutions, John Wiley \& Sons, 1966, Chapter 15, p. 295.

[6] G.S.Manning, J. Chem. Phys. 51 (1969) 924.

[7] G.S.Manning, Q. Rev. Biophys. 11 (1978) 179.

[8] G.V.Ramanathan, J. Chem. Phys. 85 (1986) 2957.

[9] D.Stigter, Biophysical J. 69 (1995) 380.

[10] D.Stigter, K.A.Dill, Biophysical J. 71 (1996) 2064.

[11] J.A.Marinsky, in W. Stumm (Ed.), Aquatic Surface Chemistry, Wiley Interscience, New York, 1987, Chapter 3, p. 49.

[12] T.Miyajima, in J. A. Marinsky and Y. Marcus (Eds.), Ion exchange and solvent extraction, Marcel Dekker, New York, 1995, Chapter 7, p. 275.

[13] M.F.Benedetti, W.H.van Riemsdijk, L.K.Koopal, Environ. Sci. Technol. 30 (1996) 1805.

[14] J.L.Garcés, F.Mas, J.Puy, J.Galceran, J.Salvador, J. Chem. Soc. Faraday Trans. 94 (1998) 2783.

[15] J.L.Garcés, F.Mas, J.Cecília, E.Companys, J.Galceran, J.Salvador, J.Puy, Phys. Chem. Chem. Phys. 4 (2002) 3764.

[16] W.H.van Riemsdijk, L.K.Koopal, in J. Buffle and H. P. van Leeuwen (Eds.), Environmental particles, Lewis Publishers, Boca Raton, 1992, Chapter 12.

[17] J.L.Garcés, F.Mas, J.Puy, J. Chem. Phys. 111 (1999) 2818.

[18] T.Miyajima, M.Mori, S.I.Ishiguro, K.H.Chung, C.H.Moon, J. Colloid Interface Sci. 184 (1996) 279.

[19] T.Miyajima, M.Mori, S.I.Ishiguro, J. Colloid Interface Sci. 187 (1997) 259.

[20] D.G.Kinniburgh, W.H.van Riemsdijk, L.K.Koopal, M.Borkovec, M.F.Benedetti, M.J.Avena, Colloids Surfaces A 151 (1999) 147.

[21] M.J.Avena, A.W.P.Vermeer, L.K.Koopal, Colloids Surfaces A 151 (1999) 213.

[22] A.W.P.Vermeer. Interaction betwen humic acid and hematite and their effects on metal ion speciation Ph.D. Thesis. Agricultural University, Wageningen, 1996.

[23] H.Ohshima, K.Fukusawa, Electrical phenomena at interfaces. Fundamentals, measurements and applications, Marcel Dekker, Inc, New York, 1998.

[24] E.Companys. Speciation of heavy metals in macromolecular systems by electroanalytical techniques Universitat de Lleida, 2003. 
[25] G.Gran, Analyst. 77 (1952) 661.

[26] J.C.M.de Wit, W.H.van Riemsdijk, M.M.Nederlof, D.G.Kinniburgh, L.K.Koopal, Anal. Chim. Acta 232 (1990) 189.

[27] J.C.M.de Wit, W.H.van Riemsdijk, L.K.Koopal, Environ. Sci. Technol. 27 (1993) 2005.

[28] M.Nagasawa, T.Murase, K.Kondo, J. Phys. Chem. 69 (1965) 4005.

[29] W.P.J.van der Drift. Ph.D. Thesis. State University Utrecht, 1975.

[30] J.L.Garcés, F.Mas, J.Cecília, J.Galceran, J.Salvador, J.Puy, Analyst. 121 (1996) 1855.

[31] C.J.Milne, D.G.Kinniburgh, E.Tipping, Environ. Sci. Technol. 35 (2001) 2049.

[32] S.E.Cabaniss, Anal. Chim. Acta 255 (1991) 23.

[33] E.B.H.Santos, V.I.Esteves, J.P.C.Rodrigues, A.C.Duarte, Anal. Chim. Acta 392 (1999) 333.

[34] P.Barak, Y.Chen, Soil Sci. 154 (1992) 184.

[35] J.D.Ritchie, E.M.Perdue, Geochim. Cosmochim. Ac. 67 (2003) 85.

[36] B.M.Bartschart, S.E.Cabaniss, F.M.M.Morel, Environ. Sci. Technol. 26 (1992) 284.

[37] C.J.Milne, D.G.Kinniburgh, J.C.M.de Wit, W.H.van Riemsdijk, L.K.Koopal, Geochim. Cosmochim. Ac. 59 (1995) 1101.

[38] E.Balnois, K.J.E.Wilkinson, J.R.Lead, J.Buffle, Environ. Sci. Technol. 33 (1999) 3911.

[39] J.Buffle, K.J.Wilkinson, S.Stoll, M.Filella, J.Zhang, Environ. Sci. Technol. 32 (1998) 2887.

[40] F.J.Stevenson, Humus Chemistry. Genesis, Composition, Reactions, John Wiley \& Sons, New York, 1994.

[41] R.Netz, H.Orland, Eur. Phys. J. 1 (2000) 203.

[42] T.J.R.Hughes, The Finite Element Method, Prentice Hall, Englewood Cliffs,N.J., 1987.

[43] W.H.Press, B.P.Flannery, S.A.Teukolsky, W.T.Vetterling, Numerical Recipes, Cambridge University Press, Cambridge, 1986. 


\section{Tables}

Table 1. Isotherms used in this work. The first column gathers the expressions for the coverage $(\theta)$ in terms of the various characteristic parameters of each isotherm; the mean $(\mu)$ and the standard deviation $(\sigma)$ in the following columns are expressed as functions of the corresponding isotherm parameters [15].

\begin{tabular}{|c|c|c|c|}
\hline Isotherm & $\theta$ & $\mu=<\log k>$ & $\left.\sigma=\sqrt{\left(<(\log k)^{2}>-\mu^{2}\right.}\right)$ \\
\hline L-F & $\frac{\left(k_{\mathrm{m}} c\right)^{\Gamma}}{1+\left(k_{\mathrm{m}} c\right)^{\Gamma}}$ & $\log k_{\mathrm{m}}$ & $\frac{\pi}{(\ln 10)} \frac{\sqrt{1-\Gamma^{2}}}{3 \Gamma^{2}}$ \\
\hline Frumkin & $\frac{K c e^{-2 \rho \theta}}{1+K c e^{-2 \rho \theta}}$ & $\log K-\frac{\rho}{(\ln 10)}$ & $\frac{\pi}{\ln 10} \sqrt{\frac{\rho^{2}}{3}+2 \rho}$ \\
\hline Uniform & $\frac{1}{2 h} \log \left(\frac{1+10^{\mathrm{h}} k_{\mathrm{m}} c}{1+10^{-\mathrm{h}} k_{\mathrm{m}} c}\right)$ & $\log k_{\mathrm{m}}$ & $\frac{h}{\sqrt{3}}$ \\
\hline
\end{tabular}

Table 2. Fitted parameters derived for proton binding to PAA by solving Poisson Boltzmann in cylindrical geometry with $r=$ $0.55 \mathrm{~nm}$.

\begin{tabular}{|c|c|c|c|c|}
\hline Isotherm & & & $\mu=\log \left(k_{\mathrm{m}} / \mathrm{M}^{-1}\right)$ & $\sigma=\left(\sqrt{\left\langle\log ^{2}\left(k_{\mathrm{m}} / \mathrm{M}^{-1}\right)\right\rangle-\mu^{2}}\right)$ \\
\hline L-F & $k_{\mathrm{m}}=(2.49 \pm 0.03) 10^{4} \mathrm{M}^{-1}$ & $m=0.840 \pm 0.006$ & $4.40 \pm 0.01$ & $0.51 \pm 0.01$ \\
\hline Frumkin & $K=(4.2 \pm 0.1) 10^{4} \mathrm{M}^{-1}$ & $\rho=0.50 \pm 0.02$ & $4.41 \pm 0.01$ & $0.45 \pm 0.01$ \\
\hline Uniform & $k_{\mathrm{m}}=(2.57 \pm 0.03) 10^{4} \mathrm{M}^{-1}$ & $h=0.78 \pm 0.01$ & $4.41 \pm 0.01$ & $0.45 \pm 0.01$ \\
\hline Mean value & & & $4.41 \pm 0.01$ & $0.48 \pm 0.03$ \\
\hline
\end{tabular}

Table 3. Fitted parameters derived for proton binding to PAA by Donnan approach and different complexation isotherms assuming the expression for $V_{\mathrm{D}}$ given in (15).

\begin{tabular}{|c|c|c|c|c|c|}
\hline Isotherm & & & $a / \mathrm{M}^{-1 / 2}$ & $\mu=\log \left(k_{\mathrm{m}} / \mathrm{M}^{-1}\right)$ & $\sigma=\left(\sqrt{\left\langle\log ^{2}\left(k_{\mathrm{m}} / \mathrm{M}^{-1}\right)\right\rangle-\mu^{2}}\right.$ \\
\hline L-F & $k_{\mathrm{m}}=(2.5 \pm 0.3) 10^{4} \mathrm{M}^{-1}$ & $m=0.84 \pm 0.02$ & $190 \pm 13$ & $4.40 \pm 0.06$ & $0.52 \pm 0.04$ \\
\hline Frumkin & $K=(4.2 \pm 0.7) 10^{4} \mathrm{M}^{-1}$ & $\rho=0.50 \pm 0.06$ & $190 \pm 13$ & $4.41 \pm 0.08$ & $0.45 \pm 0.03$ \\
\hline Uniform & $k_{\mathrm{m}}=(2.6 \pm 0.3) 10^{4} \mathrm{M}^{-1}$ & $h=0.80 \pm 0.05$ & $190 \pm 12$ & $4.41 \pm 0.05$ & $0.46 \pm 0.03$ \\
\hline Mean value & & & $190 \pm 13$ & $4.41 \pm 0.04$ & $0.49 \pm 0.04$ \\
\hline
\end{tabular}


Table 4. Parameters derived for proton binding to the purified humic acid by the Poisson-Boltzmann model (spherical geometry) with $r_{0}=3.5 \mathrm{~nm}$ and $n_{\mathrm{p}}=30$ and different complexation isotherms.

\begin{tabular}{|c|c|c|c|c|}
\hline Isotherm & & & $\mu=\log \left(k_{\mathrm{m}} / \mathrm{M}^{-1}\right)$ & $\sigma=\left(\sqrt{\left\langle\log ^{2}\left(k_{\mathrm{m}} / \mathrm{M}^{-1}\right)\right\rangle-\mu^{2}}\right)$ \\
\hline L-F & $k_{\mathrm{m}}=(1.45 \pm 0.01) 10^{4} \mathrm{M}^{-1}$ & $m=0.387 \pm 0.002$ & $4.160 \pm 0.004$ & $1.875 \pm 0.009$ \\
\hline Frumkin & $K=(8.6 \pm 0.3) 10^{5} \mathrm{M}^{-1}$ & $\rho=4.03 \pm 0.04$ & $4.18 \pm 0.02$ & $1.59 \pm 0.01$ \\
\hline Uniform & $k_{\mathrm{m}}=(1.57 \pm 0.04) 10^{4} \mathrm{M}^{-1}$ & $h=2.74 \pm 0.02$ & $4.19 \pm 0.01$ & $1.58 \pm 0.01$ \\
\hline Mean value & & & $4.18 \pm 0.01$ & $1.7 \pm 0.1$ \\
\hline
\end{tabular}

Table 5. Parameters derived for proton binding to the purified humic acid by Donnan approach and different complexation isotherms (with $\log V_{\mathrm{D}}=b(1-\log I)-1$ and $\left.b=0.43\right)$.

\begin{tabular}{|c|c|c|c|c|}
\hline Isotherm & & & $\mu=\log \left(k_{\mathrm{m}} / \mathrm{M}^{-1}\right)$ & $\sigma=\left(\sqrt{\left\langle\log ^{2}\left(k_{\mathrm{m}} / \mathrm{M}^{-1}\right)\right\rangle-\mu^{2}}\right)$ \\
\hline L-F & $k_{\mathrm{m}}=(8.40 \pm 0.10) 10^{2} \mathrm{M}^{-1}$ & $m=0.35 \pm 0.00$ & $2.92 \pm 0.01$ & $2.11 \pm 0.02$ \\
\hline Frumkin & $K=(5.3 \pm 0.3) 10^{4} \mathrm{M}^{-1}$ & $\rho=4.10 \pm 0.04$ & $2.94 \pm 0.03$ & $1.61 \pm 0.01$ \\
\hline Uniform & $k_{\mathrm{m}}=(8.88 \pm 0.12) 10^{2} \mathrm{M}^{-1}$ & $h=2.70 \pm 0.02$ & $2.95 \pm 0.01$ & $1.56 \pm 0.01$ \\
\hline Mean value & & & $2.93 \pm 0.02$ & $1.8 \pm 0.3$ \\
\hline
\end{tabular}

Table 6. Parameters derived for proton binding to the purified humic acid by Donnan approach and different complexation isotherms (assuming the expression for $V_{\mathrm{D}}$ given in (15)).

\begin{tabular}{|c|c|c|c|c|c|}
\hline Isotherm & & & $a / \mathrm{kg}(\mathrm{mol} \mathrm{L})^{-1 / 2}$ & $\mu=\log \left(k_{\mathrm{m}} / \mathrm{M}^{-1}\right)$ & $\sigma=\left(\sqrt{\left\langle\log ^{2}\left(k_{\mathrm{m}} / \mathrm{M}^{-1}\right)\right\rangle-\mu^{2}}\right.$ \\
\hline L-F & $k_{\mathrm{m}}=(1.97 \pm 0.05) 10^{4} \mathrm{M}^{-1}$ & $\Gamma=0.368 \pm 0.002$ & $0.032 \pm 0.001$ & $4.30 \pm 0.01$ & $1.99 \pm 0.01$ \\
\hline Frumkin & $K=(1.59 \pm 0.09) 10^{6} \mathrm{M}^{-1}$ & $\rho=4.22 \pm 0.04$ & $0.029 \pm 0.001$ & $4.37 \pm 0.03$ & $1.65 \pm 0.01$ \\
\hline Uniform & $k_{\mathrm{m}}=(2.4 \pm 0.1) 10^{4} \mathrm{M}^{-1}$ & $h=2.78 \pm 0.02$ & $0.028 \pm 0.001$ & $4.38 \pm 0.02$ & $1.60 \pm 0.01$ \\
\hline Mean value & & & $0.030 \pm 0.002$ & $4.34 \pm 0.04$ & $1.8 \pm 0.2$ \\
\hline
\end{tabular}




\section{Figures}

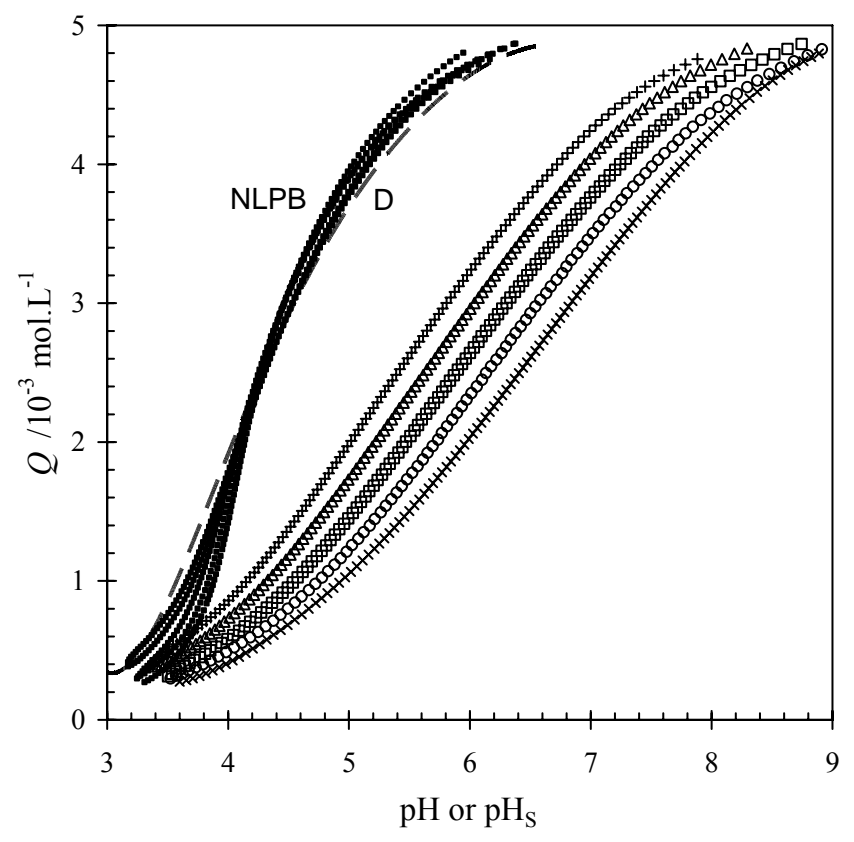

Figure 1. Experimental $Q v s \mathrm{pH}$ data of the PAA titrations (appearing on the right of the plot). Markers, $I=0.1 \mathrm{M}(+), 0.05 \mathrm{M}$ $(\Delta), 0.02 \mathrm{M}(\square), 0.01 \mathrm{M}(\mathrm{o}), 0.005 \mathrm{M}(\mathrm{x})$. "Master curves", $Q v s \mathrm{pH}_{\mathrm{S}}$, obtained solving the Poisson-Boltzmann equation in cylindrical geometry (marker $\boldsymbol{\square}$ on the left of the plot) with $r=0.55 \mathrm{~nm}$ (set of quasi-collapsed curves, where the different $I$ or different isotherms are not distinguished), and applying the Donnan model with $V_{\mathrm{D}}$ given by expression (15) (dashed line).

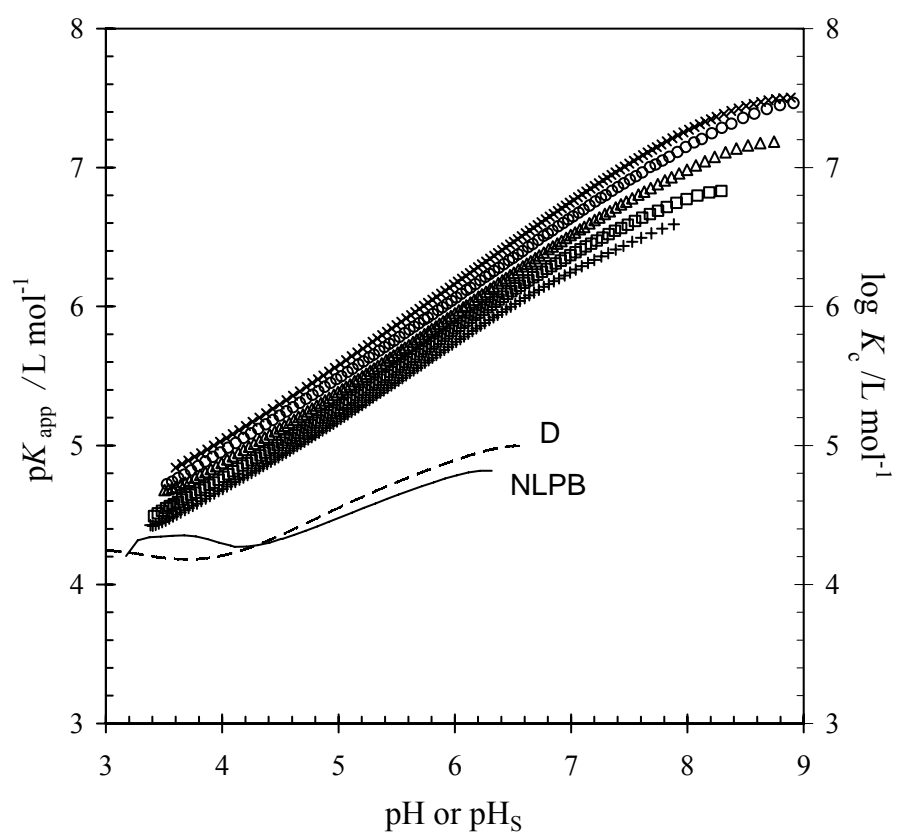

Figure 2. Experimental $\mathrm{p} K_{\text {app }} v s \mathrm{pH}$ data for the titration of PAA (markers as in Fig. 1a). "Master curves", $\log K_{\mathrm{c}} v s$ pH obtained: i) solving the Poisson-Boltzmann equation in cylindrical geometry (continuous line), and ii) through the Donnan model with the suggested expression for $V_{\mathrm{D}}(15)$ (dashed line). The master curve plotted in each case is the average of the corresponding master curves (very similar, not shown) obtained at the different $I$-values given in Fig. 1. 


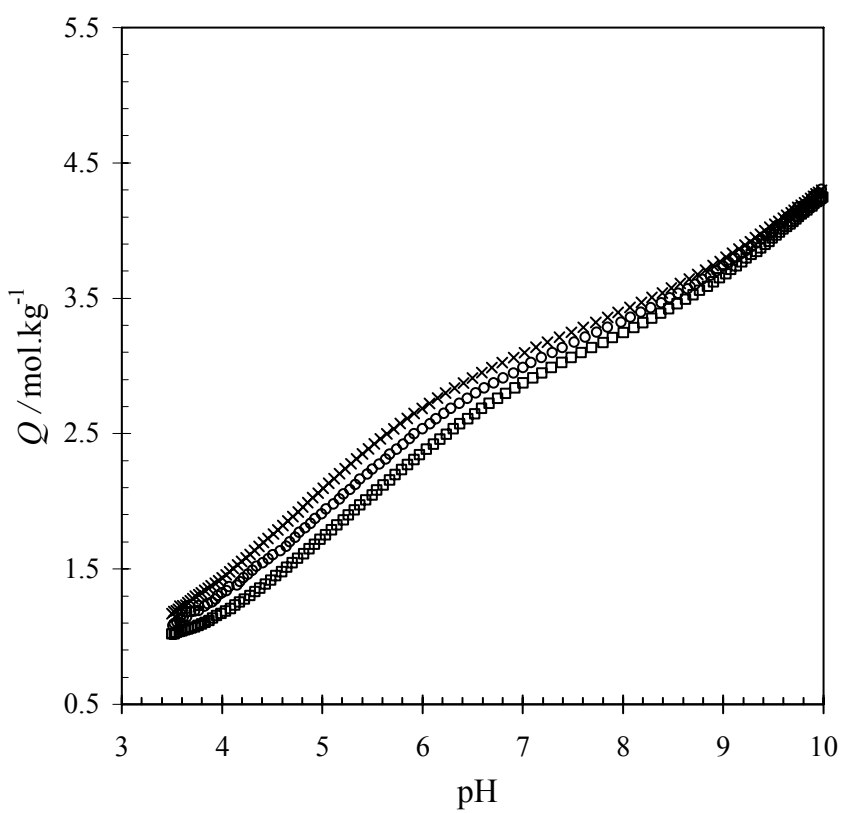

Figure 3. Proton titration curves of the purified Aldrich humic acid (markers $I=0.05 \mathrm{M}(\mathrm{x}), 0.01 \mathrm{M}(\mathrm{o}), 0.002 \mathrm{M}(\mathrm{)})$ ).

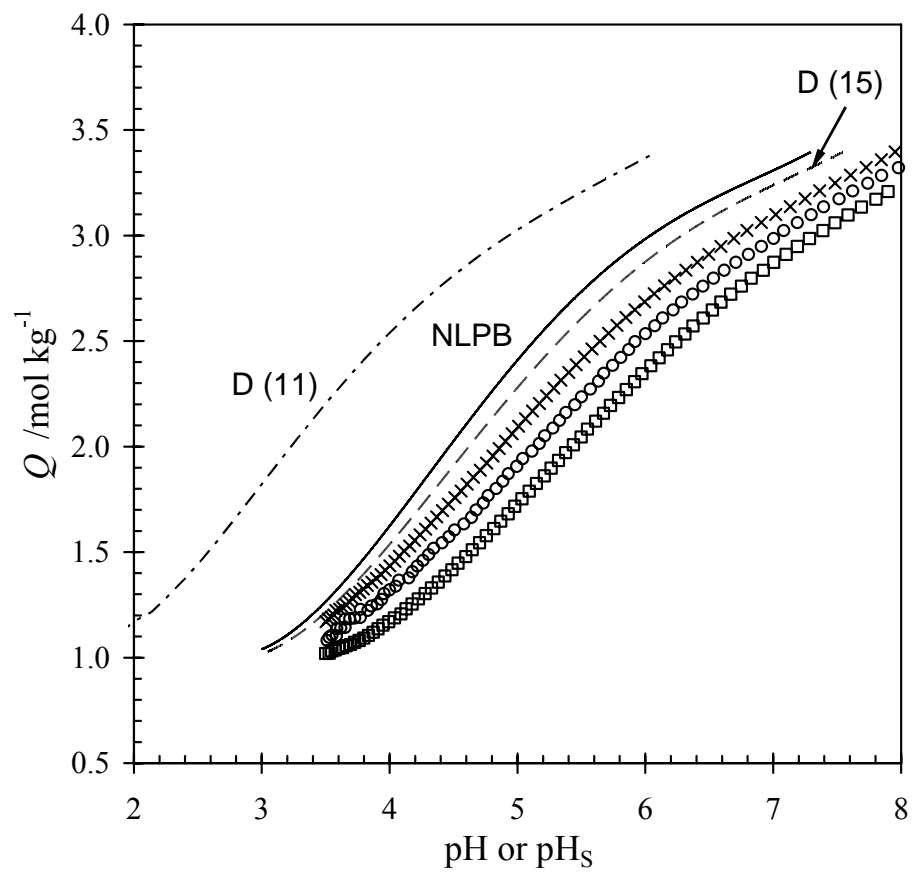

Figure 4. Experimental $Q(\mathrm{pH})$ data for the titrations of humic acid appearing on the right of the plot. Markers, $I=0.05 \mathrm{M}$ (x), $0.01 \mathrm{M}(\mathrm{o}), 0.002 \mathrm{M}(\square)$. "Master curves", $Q\left(\mathrm{pH}_{\mathrm{S}}\right)$, obtained: i) solving the Poisson-Boltzmann equation in spherical geometry (continuous line) with $r_{0}=3.5 \mathrm{~nm}$ and $\left.n_{\mathrm{p}}=30\right)$, ii) through the Donnan model with $\log V_{\mathrm{D}}=b(1-\log I)-1(b=0.43)$ (dotteddashed line), and iii) through the Donnan model with (15) (dashed line). 


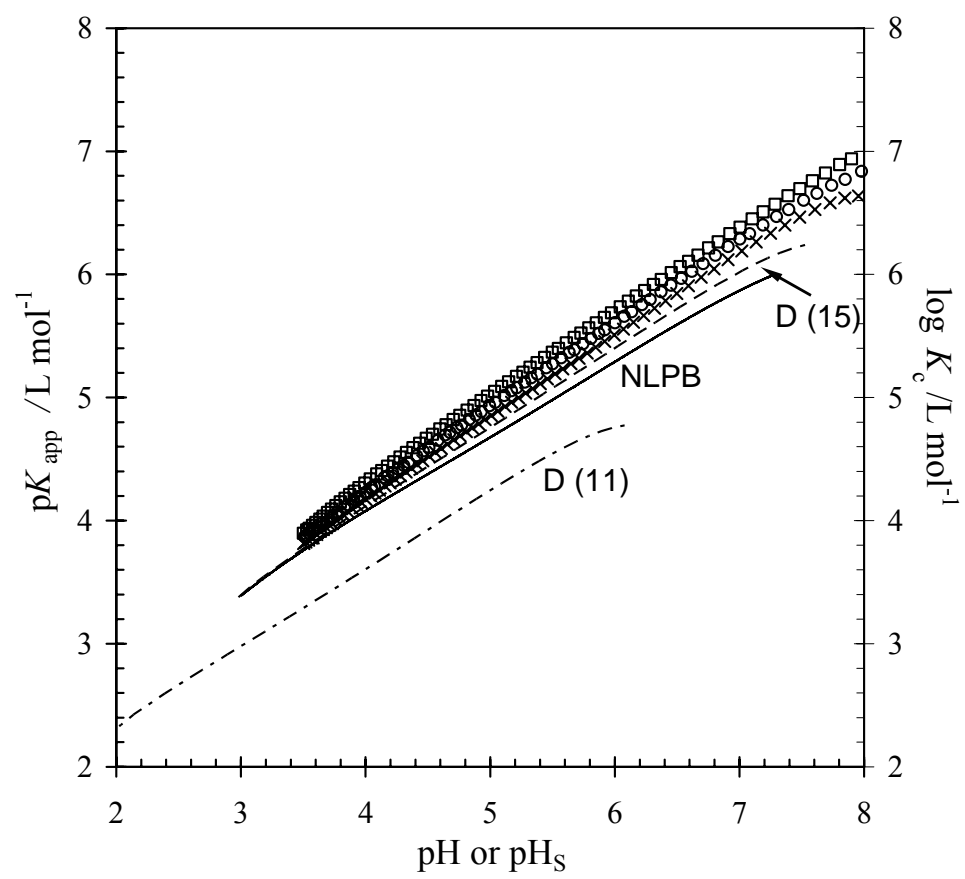

Figure 5. Experimental $\mathrm{p} K_{\text {app }}(\mathrm{pH})$ data for the titration of HA (markers as in Fig.7a). "Master curves", $\log K_{\mathrm{c}}\left(\mathrm{pH}_{\mathrm{S}}\right)$ obtained: i) solving the Poisson-Boltzmann equation in spherical geometry (continuous line), ii) through the Donnan model with $\log V_{\mathrm{D}}=b(1$ $-\log I)-1(b=0.43)$ (dotted-dashed line), and iii) through the Donnan model with (15) (dashed line). The master curve plotted in any case is the average of the corresponding master curves obtained at the different $I$-values given in Fig. 4.

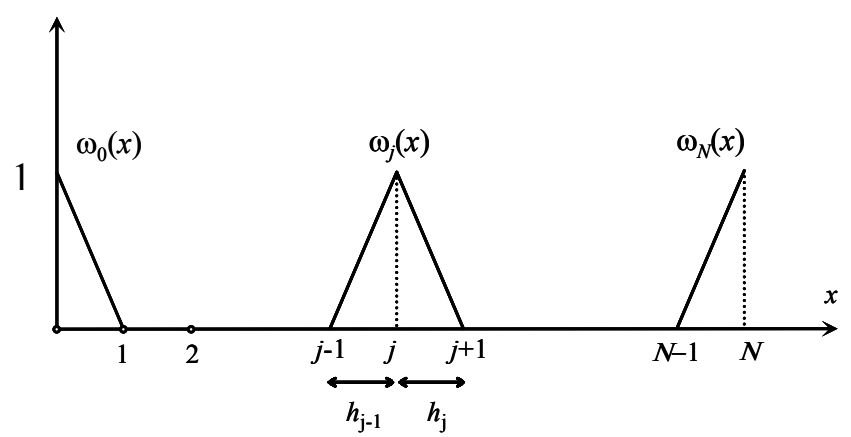

Figure 6. Mesh and base functions used to interpolate the solution function. 